\title{
Rutin attenuates isoflurane-induced neuroapoptosis via modulating JNK and p38 MAPK pathways in the hippocampi of neonatal rats
}

\author{
WEI LI ${ }^{1}$, DE-YUAN LI ${ }^{2}$, SI-MING ZHAO ${ }^{3}$, ZHE-JUN ZHENG ${ }^{2}$, JIE HU $^{2}$, ZONG-ZHE LI $^{2}$ and SHAN-BAI XIONG ${ }^{3}$ \\ ${ }^{1}$ Hubei Cooperative Innovation Center for Industrial Fermentation, Hubei University of Technology; \\ ${ }^{2}$ Department of Nutrition and Food Research Institute, Wuhan Economic College; \\ ${ }^{3}$ Department of Food Science and Technology, Huazhong Agricultural University, Wuhan, Hubei 430035, P.R. China
}

Received June 16, 2015; Accepted September 6, 2016

DOI: $10.3892 /$ etm.2017.4173

\begin{abstract}
An increasing number of infants and children undergo surgery and are exposed to anesthesia as a part of medical care each year. Isoflurane is a commonly used anesthetic in the pediatric population. However, previous studies have reported widespread isoflurane-induced neuroapoptosis and cognitive impairments in neonatal animal models, raising concerns over the administration of isoflurane in the pediatric population. The current study investigated the effects of rutin, a flavonoid, on isoflurane-induced neuroapoptosis in a neonatal rodent model. Groups of neonatal rat pups were administered rutin at doses of 10,20 or $40 \mathrm{mg} / \mathrm{kg}$ body weight from postnatal day 1 (P1) to P15. On P7, pups were exposed to $0.75 \%$ isoflurane for $6 \mathrm{~h}$. Rat pups in the control groups did not receive rutin, and did not receive anesthesia in one group. Neuroapoptosis following isoflurane exposure was determined by TUNEL assay. The expression levels of cleaved caspase-3, apoptotic pathway proteins [Bcl2-associated agonist of cell death (Bad), phospho-Bad, Bax, B-cell lymphoma 2 (Bcl-2) and $\mathrm{Bcl}-\mathrm{xL}$ and mitogen-activated protein kinases (MAPK)] signalling pathway proteins [c-Jun N-terminal kinase (JNK), phospho-JNK, extracellular-signal-regulated kinase 1/2 (ERK1/2), phosphoERK1/2, p38, phospho-p38 and phospho-c-Jun], were determined by western blot analysis. The Morris water maze test was used to assess the learning and memory of pups on P30 and P31. The present study found that rutin at the tested doses of 10,20 and $40 \mathrm{mg}$ significantly reduced $(\mathrm{P}<0.05)$ the isoflurane-induced elevation in apoptotic cell count. The expression levels of caspase-3, Bad, Bax and MAPK proteins, which were increased following isoflurane
\end{abstract}

Correspondence to: Dr De-Yuan Li, Department of Nutrition and Food Research Institute, Wuhan Economic College, 22 Luojiadun Street, Qiaokou, Wuhan, Hubei 430035, P.R. China

E-mail: lideyuan326@gmail.com

Key words: isoflurane, mitogen activated protein kinases, neonatal, neuroapoptosis, rutin treatment, were rescued by rutin treatment. Furthermore, rutin prevented the increase in Bcl-xL, Bcl-2 and phospho-Bad expression following isoflurane treatment, and enhanced the memory of the rats. Rutin provided neuroprotection against isoflurane-induced neuronal apoptosis and improved the learning and memory of rats by effectively regulating the expression levels of proteins in the MAPK pathway.

\section{Introduction}

Volatile anesthetics such as isoflurane and sevoflurane are used in pediatric surgical procedures and in imaging studies (1). Extended exposure to volatile anesthetics causes widespread neurodegeneration in the developing brains of animals, and also results in persistent learning and memory deficits (2-7). Children $<4$ years of age who have undergone surgery under general anesthesia more than once have a greater likelihood of developing cognitive disabilities and also experience difficulties in reading and learning $(8,9)$. Previous studies have raised serious concerns regarding the safety of such anesthetics, and have therefore necessitated protective strategies $(2,3,8,9)$.

Isoflurane has been reported to induce neuroapoptosis and degeneration by $\left[\mathrm{Ca}^{2+}\right]_{\mathrm{i}}$ overload $(10,11)$, which subsequently activates the mitochondrial pathways of apoptosis $(12,13)$, and has also been demonstrated to be associated with the activation of c-Jun N-terminal kinase (JNK) (4). JNK is a member of the mitogen activated protein kinases (MAPKs). MAPKs are a family of serine-threonine protein kinases that consist of three major members: Extracellular-signal-regulated kinases (ERKs), p38 MAPK and JNK. MAPK signalling pathways serve important roles in cell survival, development, neurodegeneration, pain and inflammation under normal and pathological conditions (14-17). The JNK signalling cascade involves mediating neuronal apoptosis through the regulation of Bcl-2 family members and activation of c-Jun (18), resulting in indirect transcriptional regulation of the $\mathrm{Bcl}-2$ family members (19), including downregulation of the anti-apoptotic proteins $(19,20)$.

Recent studies have revealed that the involvement of MAPK cascades in anesthetics-induced neurotoxicity. Li et al (7) reported the involvement of the JNK pathway in 
isoflurane-induced neurodegeneration in the hippocampi of neonatal rats. Furthermore, N-stearoyl-l-tyrosine was reported to protect the developing brain against sevoflurane-induced neurotoxicity via the ERK1/2 signalling pathway (21). Finally, Sanders et al (22) reported that dexmedetomidine upregulated ERK1/2 in brain tissues of neonatal rats exposed to isoflurane.

Rutin is a flavonoid glycoside found in numerous plants including buckwheat, limes, oranges, grapefruit and berries. Numerous pharmacological properties of rutin, including antioxidant (23), anti-inflammatory (24) and hypolipidemic properties (25) have been reported. The present study endeavoured to investigate the role of rutin in terms of providing neuroprotection against isoflurane-induced neuroapoptosis in the hippocampi of neonatal rats through the modulation of MAPK cascades.

\section{Materials and methods}

Reagents and chemicals. Isoflurane $(0.75 \%)$ and rutin were purchased from Sigma-Aldrich (Merck Millipore, Darmstadt, Germany). Antibodies against cleaved caspase-3, Bcl2-associated agonist of cell death (Bad), phospho-Bad, Bcl-xL, Bax, $\beta$-actin, JNK, phospho-JNK, phospho-c-Jun, ERK1/2, phospho-ERK1/2, p38 and phospho-p38 were purchased from Cell Signalling Technology (Danvers, MA, USA). All the chemicals used in the current study were of analytical grade and purchased from Sigma-Aldrich, unless otherwise specified.

Animals. The present study was approved (approval no. HAU24677244) by the Animal Care and Ethical Committee of Huazhong Agriculture University (Wuhan, China) and was performed in accordance with the National Institutes of Health Guide for the Use of Laboratory Animals (26). A total of 12 pregnant female Sprague-Dawley rats (Guangdong Medical Laboratory Animal Center, Guandong, China) were used in this study, and were maintained in an environment with a $12 \mathrm{~h}$ light/dark cycle and a room temperature of $22 \pm 1^{\circ} \mathrm{C}$ (humidity, 55-60\%). Animals were provided with ad libitum access to water and food and were housed individually in separate cages and monitored closely from the day of birth [postnatal day 0 (P0)]. Pups were then carefully maintained with their littermates in a standard environment, as outlined, with a $12 \mathrm{~h}$ light/dark cycle and free access to water. A total of 60 pups ( $n=12 /$ group) weighing 18-28 g were used for the experiments. Pups in the treatment groups were administered rutin [10, 20 or $40 \mathrm{mg} / \mathrm{kg}$ body weight (b.wt)] orally, once every day from P1, and treatment was continued until P15 in addition to a standard diet. Control group pups did not receive rutin or isoflurane. Separate group of rat pups that were exposed only to isoflurane served as the anesthetic control.

Exposure to anesthesia. A total of 60 pups (n=12/group) were assigned into groups as follows: Group 1, control pups, not treated with anesthetic or rutin; group 2, anesthetic control, exposed to isoflurane $(0.75 \%)$ for $6 \mathrm{~h}(7,27)$ and not treated with rutin; group 3, treated with $10 \mathrm{mg} / \mathrm{kg}$ rutin and exposed to isoflurane on P7; group 4, treated with $20 \mathrm{mg} / \mathrm{kg}$ rutin and exposed to isoflurane on P7; and group 5, treated with $40 \mathrm{mg} / \mathrm{kg}$ rutin and exposed to isoflurane on $\mathrm{P} 7$. On $\mathrm{P} 7,1 \mathrm{~h}$ prior to the delivery of isoflurane, rutin was administrated. At the end of the 6th hour of anesthetic exposure, six rat pups $(n=6)$ from each experimental group were sacrificed and their brains were excised. For sacrifice, within $1 \mathrm{~h}$ of anesthesia, pups were perfused transcardially with ice-cold saline followed by $4 \%$ paraformaldehyde in $0.1 \mathrm{M}$ phosphate buffer The hippocampi of the rat pups were then used for protein expression analysis by western blotting and TUNEL assay. The remaining rats $(n=6)$ continued to receive rutin supplementation until P15, and were then maintained according to the standard experimental conditions until P31. Rats were subjected to memory and learning studies via Morris Water Maze tests.

TUNEL assay. TUNEL studies were performed as described previously by Li et al (7). Briefly, rat pups that were anesthetized with isoflurane were perfused transcardially with ice-cold saline, followed by $4 \%$ paraformaldehyde in $0.1 \mathrm{M}$ phosphate buffer. The brain tissues were harvested and post-fixed in formaldehyde for $48 \mathrm{~h}$ at $4^{\circ} \mathrm{C}$, embedded in paraffin and sectioned ( $5 \mu \mathrm{m}$ thickness). TUNEL positive cells were determined using the Dead End fluorometric TUNEL System kit (Promega Corporation, Madision, WI, USA) and positive cell counts in the hippocampal CA1, CA3 and DG regions were analyzed with an inverted Eclipse Ti2 microscope and NIS-Elements BR imaging processing and analysis software (Nikon Corporation, Tokyo, Japan).

Western blot analysis. Western blot analysis was performed to assess the expression levels of various proteins following isoflurane exposure and rutin treatment. Western blot analysis was performed as previously described by Li et al $(6,7)$. Briefly, protein concentrations were determined using a BCA protein assay (Bio-Rad Laboratories, Inc., Hercules, CA, USA). Each of the protein samples $(60 \mu \mathrm{g})$ was separated by $12 \%$ polyacrylamide gel electrophoresis, blotted on a nitrocellulose membrane and hybridized using the following antibodies: Anti-Bad (1:1,000; 9268), anti-phospho-Bad (1:1,000; 5284), anti-Bcl-xL (1:2,000; 2764), anti-cleaved caspase-3 (1:2,000; 9661), anti-Bax (1:1,000; 5023), anti-Bcl-2 (15071; 1:1,000), anti-ERK1/2 (1:1,000; 9102), anti-phospho-ERK1/2 (1:1,000; 9101), anti-JNK (1:1,000), anti-phospho-JNK (1:1,000; 9255), anti-phospho-c-Jun $(1: 1,000 ; 3270)$, anti- $\beta$-actin $(1: 2,000$; $3700)$, anti-p38 (1:1,000; 8690) and phospho-p38 (1:1,000; 4511). The immunoreactive bands were observed using an ECL detection kit (RPN2232) and images were scanned using Image Master 2D Platinum 7.0 scanner (both GE Healthcare, Pittsburgh, PA USA). Images were analyzed by ImageQuant TL software v2003.03 (version .8.3; GE Healthcare). The band signals of the proteins were normalized relative to $\beta$-actin bands.

\section{Memory and learning studies}

Morris water maze test. To assess the effects of rutin on isoflurane-induced alterations in memory and learning, spatial reference memory and learning assessments, including the Morris water maze test, were performed as previously described by Li et al (28).

The rat pups exposed to isoflurane and/or rutin were trained in the Morris water maze for 4 days between P26 and P29. A platform of $\sim 10 \mathrm{~cm}$ in diameter was submerged in a circular pool (200 $\mathrm{cm}$ in diameter, $60 \mathrm{~cm}$ depth) filled with 


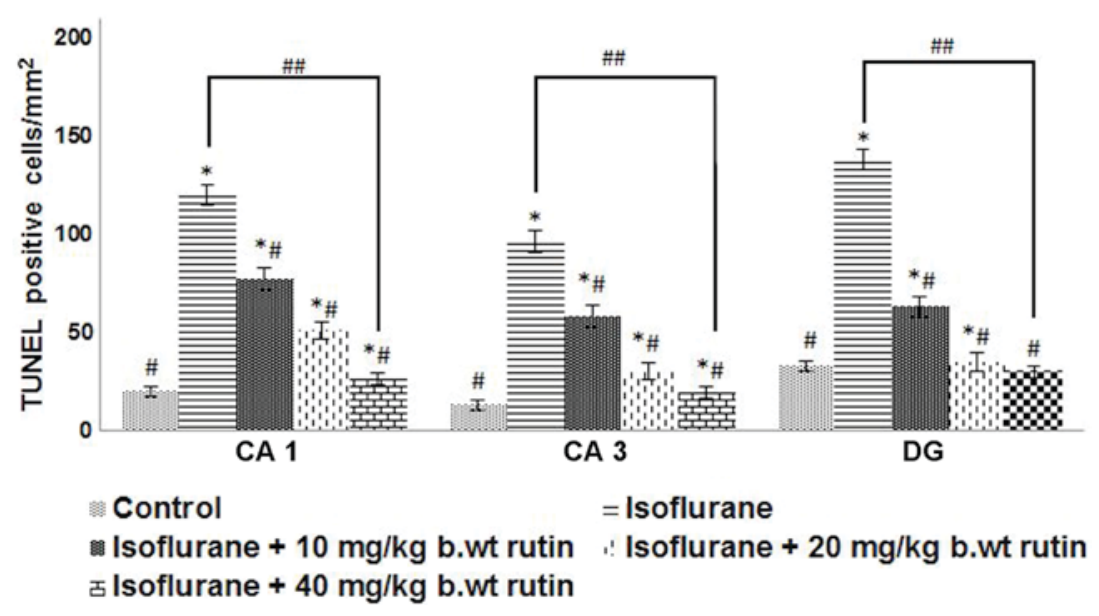

Figure 1. Effects of rutin on isoflurane-induced neuroapoptosis. Rutin reduced the apoptotic cell count following isoflurane exposure. Values are reported as the mean \pm standard deviation, $\mathrm{n}=6 .{ }^{*} \mathrm{P}<0.05$ vs. control, ${ }^{\#} \mathrm{P}<0.05$ vs. isoflurane only and ${ }^{\# \#} \mathrm{P}<0.05$, as determined by one-way analysis of variance.

warm water $\left(23 \pm 2^{\circ} \mathrm{C}\right)$. The rats were subjected to 2 sessions per day. Animals were allowed $60 \mathrm{sec}$ to locate the hidden platform, and if unable to locate it within $60 \mathrm{sec}$, they were gently guided. All trials and swim paths were recorded with ANY-maze Video Tracking system (Stoelting Co., Wood Dale, IL, USA), which measured the time taken (latency) to find the platform(s), as well as other behavioral information obtained during the spatial reference memory test. Subsequent to the trials, each rat was placed in a holding cage under an infrared heat lamp prior to being returned to its regular cage.

Cued trials. Cued trials were conducted on P31 to assess any non-cognitive performance impairments such as visual impairments and/or swimming difficulties. The pool was covered with a white cloth to conceal the visual cues. The rats undertook four trials per day. In each trial, they were placed in a particular section of the swimming pool towards the wall and were then allowed to swim to a platform with a rod that served as a cue. The rod was placed $20 \mathrm{~cm}$ above water level in a random position in any of the four quadrants of the swimming pool. The rat pups were given $60 \mathrm{sec}$ to locate the platform and $30 \mathrm{sec}$ to sit on the platform, after which they were removed from the pool. The rats that failed to locate a platform within $60 \mathrm{sec}$ were gently guided toward it and allowed to remain there for $30 \mathrm{sec}$. The time taken by each rat to reach the cued platform was recorded and analyzed.

Place trials. Subsequent to the cued trials, the white curtains that surrounded the pool were removed and the same rats were assessed in place trials to determine their ability to understand the spatial relationship between distance cues and the escape platform (no cue rod), that was placed in one of the four quadrants and remained in the same position for all place trials. The starting points were random for each rat. The time taken to reach the platform was recorded.

Probe trials. In order to assess the memory of rat pups, probe trials were conducted $24 \mathrm{~h}$ after place trials. The submerged platform was removed and the rats were placed in the opposite quadrant and allowed to swim for $60 \mathrm{sec}$. The time each rat spent in each quadrant whilst attempting to locate the platform was recorded. The data are expressed as the percentage of time spent by the animals in each of the quadrants.
Statistical analysis. All values are presented as the mean \pm standard deviation of three or six individual experiments. Multiple group comparisons were performed using one-way analysis of variance, followed by Duncan's multiple range test. The analysis was performed using SPSS statistical software (version 17.0; SPSS, Inc., Chicago, IL, USA).P<0.05 was considered to indicate a statistically significant difference.

\section{Results}

Rutin treatment reduces isoflurane-induced neuroapoptosis. Isoflurane, a commonly used volatile anesthetic, induces neuroapoptosis and long-term cognitive dysfunction in developing animals $(4,5,12)$. In the present study, apoptosis was evaluated by TUNEL assay following isoflurane exposure in P7 rat pups. The delivery of $6 \mathrm{~h}$ of isoflurane exposure significantly raised the proportion of TUNEL-positive cells in CA1, CA3 and in the dentate gyrus (DG) regions $(\mathrm{P}<0.05)$. The increases were more pronounced in the DG region when compared with the CA1 and CA3 regions. Rutin $(10,20$ or $40 \mathrm{mg} / \mathrm{kg}$ b.wt) pre-treatment significantly reduced $(\mathrm{P}<0.05)$ the TUNEL-positive cell counts in a dose-dependent manner, as compared to the isoflurane-only group (Fig. 1).

Rutin regulates the expression levels of apoptotic cascade proteins. To further assess apoptosis, quantification of cleaved caspase-3 protein expression was performed by western blot analysis. Endogenous levels of activated caspase-3 are a known and useful marker of neuronal apoptosis (29). Caspase-3 is a key cell death marker and apoptosis effector enzyme $(29,30)$. The current study revealed that isoflurane significantly induced caspase- 3 activation ( $\mathrm{P}<0.05$; Fig. $2 \mathrm{~A}$ and $\mathrm{B})$. However, rutin significantly downregulated $(\mathrm{P}<0.05)$ caspase-3 expression levels, with the $40 \mathrm{mg}$ dose presenting a more marked reduction compared with the isoflurane group. The lower dose of rutin (10 mg) reduced caspase-3 expression levels; however the reduction was not statistically significant.

Furthermore, isoflurane treatment alone resulted in the decreased expression of $\mathrm{Bcl}-\mathrm{xL}$ and $\mathrm{Bcl}-2$, the anti-apoptotic proteins in the hippocampi of $\mathrm{P} 7$ rats, but upregulated the expression of Bax, a protein that promotes apoptosis by 
A
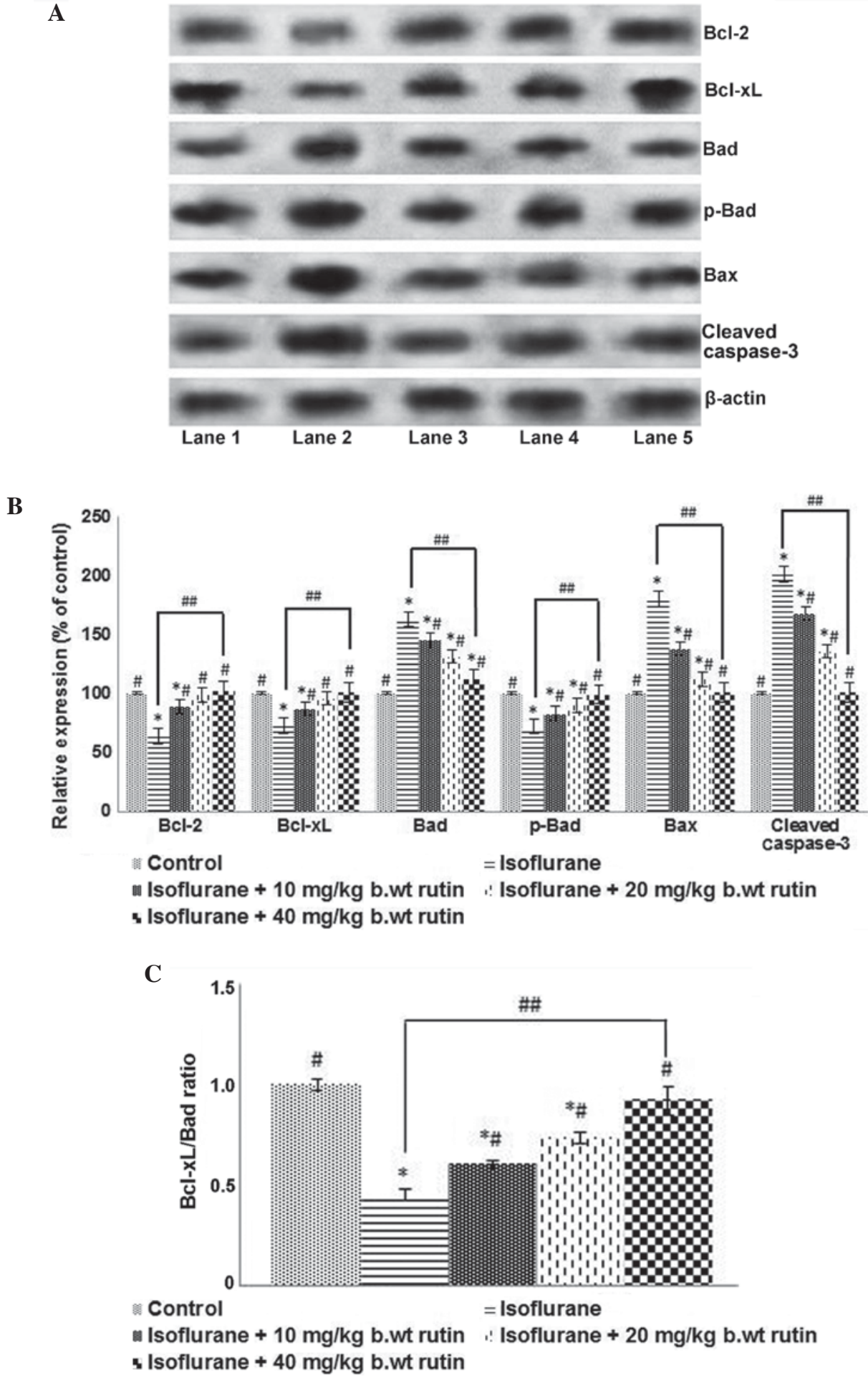

Figure 2. Effects of rutin on the expression levels of proteins in the apoptotic pathway. (A) Western blot analysis to determine the expression levels of apoptotic pathway proteins. Lane 1: Control, Lane 2: Isoflurane, Lane 3: Isoflurane+10 mg rutin, Lane 4: Isoflurane+20 mg rutin, Lane 5: Isoflurane+40 mg rutin. (B) Relative expression of apoptotic cascade proteins. Quantification of the western blot analysis indicated that isoflurane increased the expression levels of caspase-3, Bax and total Bad, and decreased Bcl-2 and Bcl-xL expression levels. (C) Rutin significantly rescued the altered expression levels of the proteins, increasing the $\mathrm{Bcl}-\mathrm{xL} / \mathrm{Bad}$ ratio $(\mathrm{C})$. Values are presented as the mean \pm standard deviation, $\mathrm{n}=3$. ${ }^{\mathrm{N}} \mathrm{P}<0.05$ vs. control, ${ }^{\#} \mathrm{P}<0.05$ vs. isoflurane only and ${ }^{\# \# /} \mathrm{P}<0.05$, as determined by one-way analysis of variance. Bcl, B-cell lymphoma, B-cell lymphoma; Bad, Bcl2-associated agonist of cell death.

binding to and antagonizing the Bcl-2 protein (20). In the present study, the increase in Bax expression correlated with the downregulation of Bcl-2, supporting this conclusion. Isoflurane however, decreased phospho-Bad expression levels, yet the total expression levels of Bad increased compared with the control group. A significant decrease in the Bcl-xL/Bad ratio was observed following isoflurane exposure compared with the control group ( $\mathrm{P}<0.05$; Fig. $2 \mathrm{C})$. Rutin at all the doses significantly ameliorated $(\mathrm{P}<0.05)$ the alteration in expression levels of the studied proteins (Fig. $2 \mathrm{~B}$ and C). Rutin significantly upregulated $(\mathrm{P}<0.05) \mathrm{Bcl}-\mathrm{xL}$ and $\mathrm{Bcl}-2$ expression levels and downregulated Bax expression levels compared 

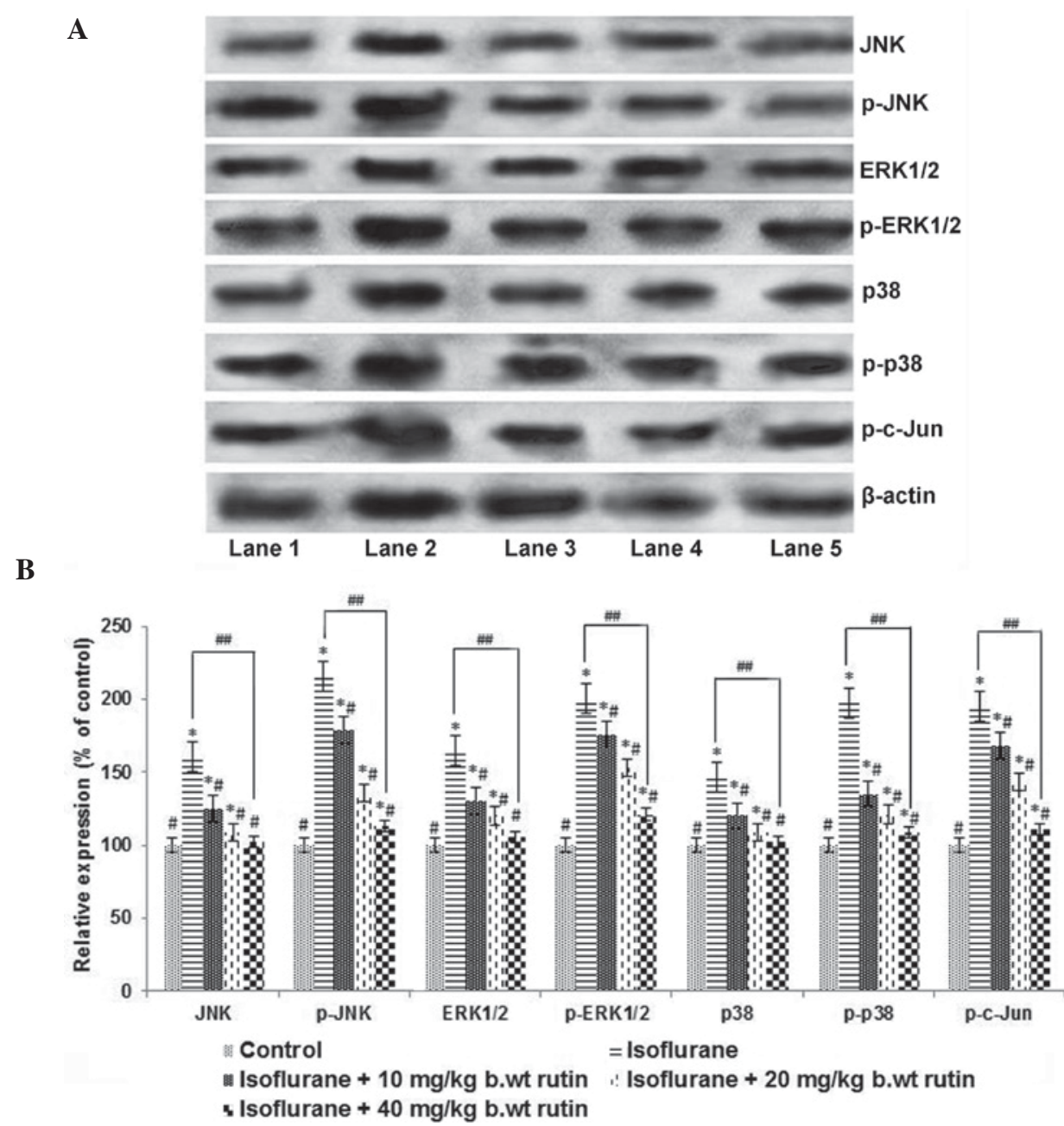

Figure 3. Rutin modulates the MAPK signalling cascade. (A) Western blot analysis to determine the expression levels of proteins in the MAPK signalling cascade. Lane 1: Control, Lane 2: Isoflurane, Lane 3: Isoflurane+10 mg rutin, Lane 4: Isoflurane+20 mg rutin, Lane 5: Isoflurane+40 mg rutin) (B) Relative expression levels of signal cascade proteins. The significant upregulation in the expression levels of MAPK proteins following $6 \mathrm{~h}$ of isoflurane exposure was effectively modulated by rutin $(10,20$ or $40 \mathrm{mg})$. Values are presented as the mean \pm standard deviation, $\mathrm{n}=3 .{ }^{*} \mathrm{P}<0.05$ vs. control, ${ }^{\prime \prime} \mathrm{P}<0.05$ vs. isoflurane only and ${ }^{\# \#} \mathrm{P}<0.05$, as determined by one-way analysis of variance. JNK, c-Jun $\mathrm{N}$-terminal kinase; ERK, extracellular-signal-regulated kinase.

with the isoflurane group. The phosphorylation of Bad has an anti-apoptotic effect, whereas its de-phosphorylation is pro-apoptotic (20). The increased levels of total Bad observed following isoflurane exposure were significantly reduced by treatment with rutin $(\mathrm{P}<0.05)$, suggesting the effectiveness of rutin for inhibiting isoflurane-induced neuroapoptosis.

Neuroprotection by rutin involves MAPK signalling cascades. To further evaluate the molecular effects of rutin on MAPK pathway proteins, the expression levels of major MAPK proteins, including JNK, ERK and p38, were analyzed by western blotting (Fig. 3A). Exposure to isoflurane for $6 \mathrm{~h}$ caused a significant increase in the phosphorylated JNK, ERK1/2 and p38 levels $(\mathrm{P}<0.05$; Fig. 3B). The expression levels of total JNK, ERK1/2 and p38 were also significantly increased $(\mathrm{P}<0.05)$. However, rutin exposure resulted in significant reductions $(\mathrm{P}<0.05)$ in the elevated levels of phospho-JNK and phospho-c-Jun compared with the isoflurane-treated group. The administration of $40 \mathrm{mg} / \mathrm{kg}$ b.wt rutin caused a significant suppression $(\mathrm{P}<0.05)$ in the phosphorylated forms of ERK1/2 and $\mathrm{p} 38$ in a dose-dependent manner. The administration of $40 \mathrm{mg}$ rutin resulted in similar protein expression levels to those of pups in the control group that were not exposed to anesthesia, which is suggestive of its protective capacity against isoflurane-induced alterations of protein expression (Fig. 3).

Effects of rutin on the behavior and memory of rats exposed to inhalation anesthesia. To evaluate the effect of neonatal exposure to isoflurane on potential learning and memory deficits, animals were subjected to the Morris water maze test, the main test used to assess spatial learning and memory (31). The rat pups exposed to isoflurane anesthesia were trained to explore the swimming pool and to reach the platform. The escape latency, which is the duration each rat takes to reach the submerged platform, was recorded. The latency was observed to decrease with each training session for all rats irrespective of whether they had been administered isoflurane and rutin or isoflurane alone (Fig. 4). The escape latencies of rutin administered groups were found to be similar to control values.

Cued trials were conducted on P31 to evaluate the swimming and visual abilities of the rat pups. The rats exposed to isoflurane anesthesia took significantly longer to reach the submerged platform compared with pups that received 


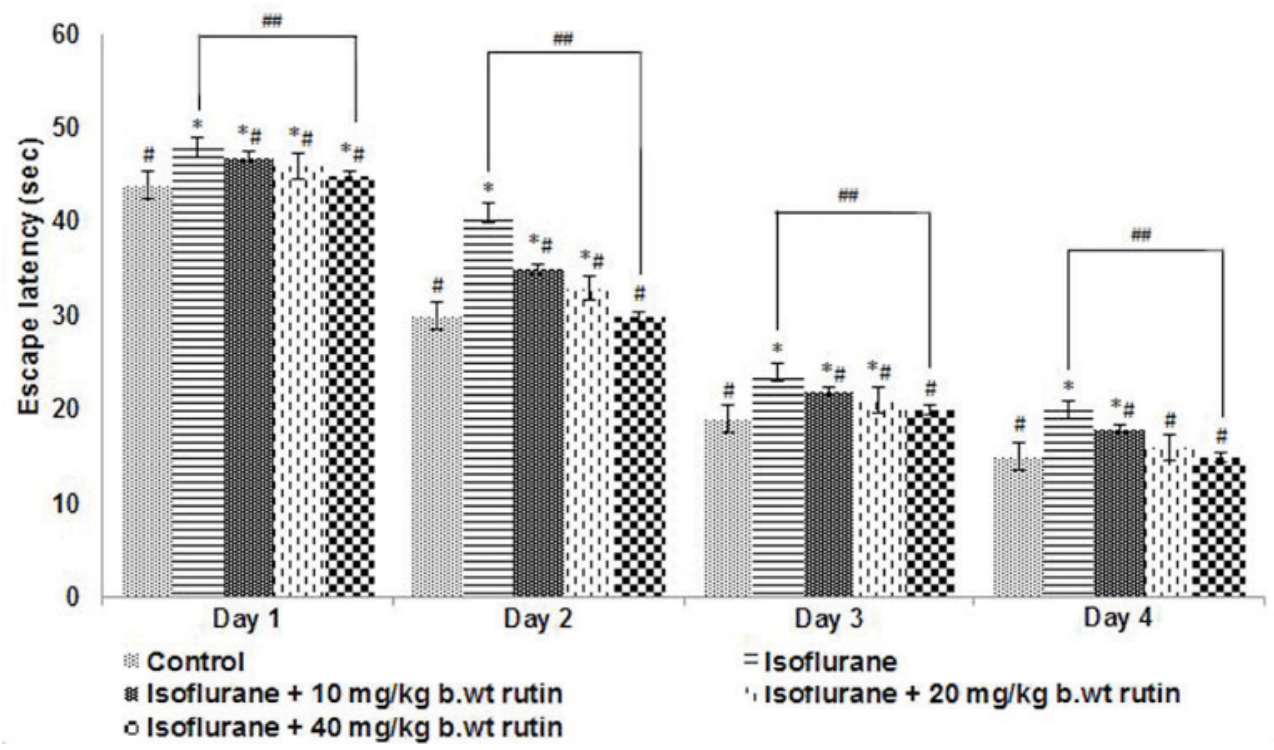

Figure 4. Escape latency of rat pups following exposure to isoflurane anesthesia on P7. On P30 rat pups were trained to reach the submerged platform. The escape latencies of pups following exposure to isoflurane alone was slightly longer compared with the other groups. Day 1 refers to the first day of training (P26). Values are represented as mean \pm standard deviation, $n=6$. ${ }^{*} \mathrm{P}<0.05$ vs. control, ${ }^{*} \mathrm{P}<0.05$ vs. isoflurane only and ${ }^{\# \#} \mathrm{P}<0.05$, as determined by one-way analysis of variance. P, postnatal day.

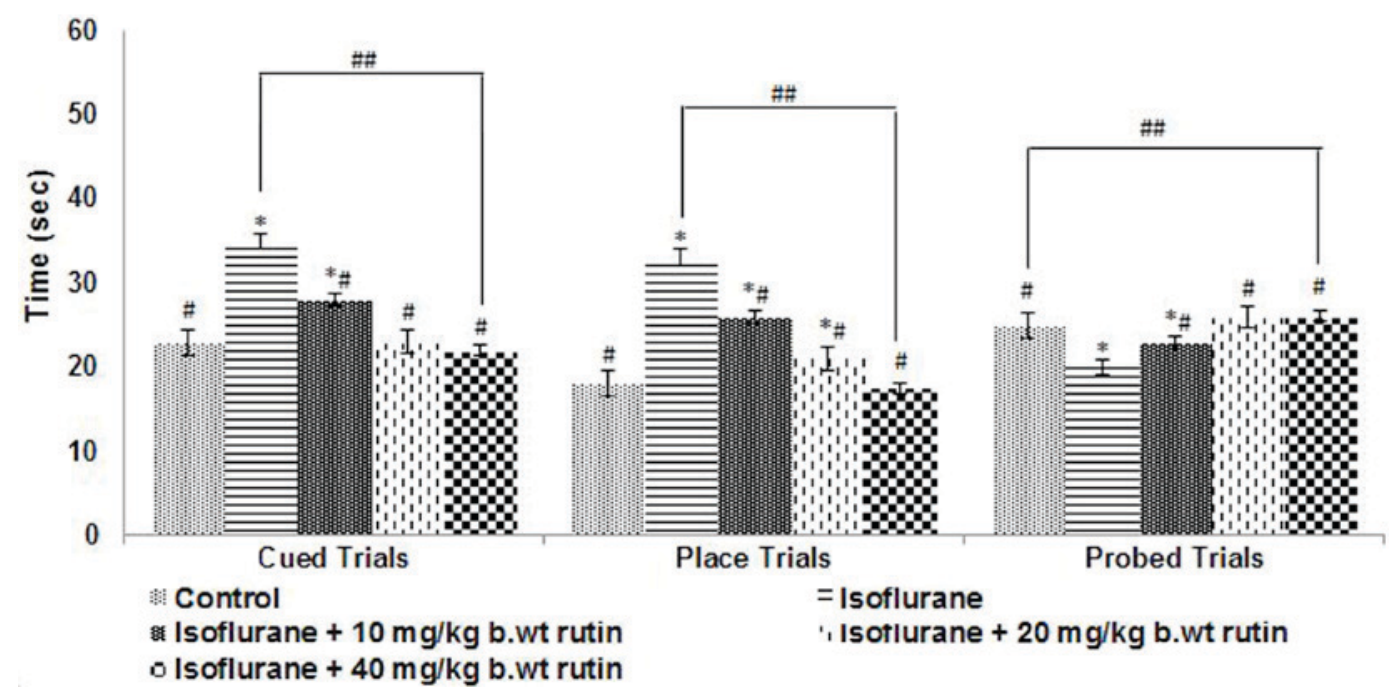

Figure 5. Learning and memory of the rat pups following anesthesia exposure on $\mathrm{P} 7$ as determined by cued, place and probe trials using the Morris water maze. On P30 and P31, rats were assessed for their learning and working memory capacities. Rutin administration markedly improved the performance of the rats. Values are represented as mean \pm standard deviation, $\mathrm{n}=6$. ${ }^{*} \mathrm{P}<0.05$ vs. control, ${ }^{,} \mathrm{P}<0.05$ vs. isoflurane only and ${ }^{\# \#} \mathrm{P}<0.05$, as determined by one-way analysis of variance.

no anesthesia $(\mathrm{P}<0.05$; Fig. 5). Rutin supplementation was observed to significantly improve the performance of the rats $(\mathrm{P}<0.05)$. The animals that received rutin were able to reach the platform quicker compared with the rats that received isoflurane alone. Furthermore, the rats that received the higher dose of rutin $(40 \mathrm{mg} / \mathrm{kg} \mathrm{b} . \mathrm{wt})$ reached the platform in a shorter time compared with those that received lower doses in the cued and place trials (Fig. 5). Place and probe trials were performed to assess the ability of the rat pups to learn and remember the location of a new platform (Fig. 5). In place trials, the rats that received 20 and $40 \mathrm{mg} / \mathrm{kg}$ b.wt rutin exhibited a significant improvement in performance $(\mathrm{P}<0.05)$, as compared with the isoflurane-only group. The rutin $(20$ and $40 \mathrm{mg}$ )-treated rats reached the platform quicker. The
$10 \mathrm{mg} / \mathrm{kg}$ b.wt dose did not elicit an enhancement in the performance of the rats.

The memory retention following exposure to isoflurane on P7 was assessed by removing the platform from the pool. Isoflurane exposure had a significantly detrimental impact on the memory of the rats compared with the control group $(\mathrm{P}<0.05$; Fig. 5), as determined by the time the animals spent in the target quadrant. The rats exposed to isoflurane spent less time in the target quadrant compared with the control group, which was indicative of the memory and learning deficits caused by isoflurane. The rats that were administered rutin spent more time on the target quadrant searching for the platform compared with the isoflurane group $(\mathrm{P}<0.05)$, indicating that there was an improvement in the memory of 
rat pups with $40 \mathrm{mg} / \mathrm{kg}$ b.wt dose of rutin compared wuth rat pups who received isoflurane alone.

\section{Discussion}

Previous studies have demonstrated the potential detrimental effects of anesthetic exposure to neonatal animals in terms of causing neurohistopathological changes and long-term behavior deficits and cognitive dysfunctions $(6,32,33)$. Apoptotic cell death is an essential part of normal brain development and maturation, leading to the elimination of $\sim 50-70 \%$ of neurons and progenitor cells during the development of the central nervous system $(34,35)$. However, during pathological processes such as hypoxia-ischemia, an absence of neurotrophic factors, or prolonged exposure to anesthetic, the rate of apoptosis surpasses the normal apoptotic rate $(36,37)$.

Isoflurane, a commonly used volatile anesthetic, has been reported to induce neurodegeneration in the developing brain of animal models $(2,4,5,7)$. The present study focused on an analysis of the effects of flavonoid rutin on isoflurane-induced neurotoxicity.

The present study demonstrated that there was an increased level of apoptosis in the brain tissues of neonatal rats exposed to $0.75 \%$ isoflurane for $6 \mathrm{~h}$. Although anesthetic-induced neurotoxicity is widespread throughout brain tissue, the current study observed the effects on the hippocampal region, as previous reports have suggested that hippocampal lesions following isoflurane exposure result in abnormal behavioral responses in neonatal rats $(2,3,5,38)$. Increased apoptotic cell counts that were observed in the CA1, CA3 and DG regions of the hippocampus following isoflurane exposure were significantly reduced with rutin treatment, demonstrating the neuroprotective effects of rutin. Previous studies have suggested that neurogenesis in the DG region is important to hippocampus-dependent episodic learning and memory, and any interference in the process may result in impaired hippocampal learning $(39,40)$.

Furthermore, the raised cleaved caspase- 3 expression was found to be consistent with TUNEL-positive cell counts as a result of anesthetic exposure. Caspase-3 serves a vital role in the apoptotic pathway (41), and in previous studies, cleaved caspase-3 expression levels were used as a marker of apoptotic cell death $(2,4,5,33)$. Rutin effectively downregulated the expression levels of activated caspase-3, in addition to the apoptotic cell count. Furthermore, the expression levels of Bax protein were upregulated following exposure to isoflurane. The anti-apoptotic protein Bcl-xL is widely expressed in the central nervous system, which enhances cell survival by maintaining mitochondrial membrane integrity and inhibits the release of cytochrome $c$ (42).

The Bcl-2 gene family encodes proteins that regulate the apoptosis program. The Bcl-2 family includes the pro-apoptotic (Bax and Bak) and anti-apoptotic (Bcl-2 and Bcl-xL) members (20). The delicate balance of the Bcl-2 family determines the release of cytochrome $c$, in addition to the subsequent activation of the caspase cascade, leading to apoptosis $(19,20)$. In the present study, isoflurane decreased the expression levels of Bcl-2, Bcl-xL and phospho-Bad. However, the elevated levels of total Bad observed following isoflurane exposure may have caused the decrease in the Bcl-xL/Bad ratio. The current results demonstrated that rutin effectively reversed the isoflurane-induced downregulation of $\mathrm{Bcl}-2, \mathrm{Bcl}-\mathrm{xL}$ and phospho-Bad, and improved the Bcl-xL/Bad ratio, which may contribute to the stabilization of the inner mitochondrial membrane, and thus inhibit isoflurane-induced neuroapoptosis.

The MAPK pathway has been reported to be involved in anesthetic-induced neurodegeneration $(7,21)$. The JNK signalling pathway is involved in neuronal apoptosis triggered by several brain injury stimuli, such as ischemic reperfusion injury (43-45). Previous studies have demonstrated that the activation of JNK signalling is involved in isoflurane-induced neuronal apoptosis (7). Activated JNK phosphorylates a nuclear substrate, the transcription factor c-Jun, which results in an increase of activator protein-1 transcription activity to modulate the transcription of genes associated with apoptosis. By contrast, activated JNK regulates the activation of non-nuclear substrates, including Bcl-2 family members $(43,45)$.

The present results indicated that rutin pretreatment inactivated the JNK nuclear pathway by reducing the isoflurane-induced increase in the phosphorylation of JNK and transcription factor c-Jun. Rutin also inhibited the JNK non-nuclear pathway by preventing the isoflurane-induced downregulation of $\mathrm{Bcl}-2$, and increasing the expression levels of Bax. Furthermore, rutin caused a marked decrease in the expression levels of phospho-ERK1/2 that had been upregulated following exposure to isoflurane, which was similar to the protective effect observed with dexmedetomidine (22). Zheng and Zuo (46) reported that isoflurane activated p38 MAPK in the brains of rats. The current study reported an increase in the activation of $\mathrm{p} 38$ following the exposure of neonatal rats pups to isoflurane on P7. However, rutin decreased phospho-p38 expression levels in a dose-dependent manner. Rutin $(40 \mathrm{mg} / \mathrm{kg} \mathrm{b}$. wt) resulted in the regulation of the expression patterns of the MAPK pathway proteins compared with lower doses. These results suggest the potential involvement of the MAPK signalling pathway in both isoflurane-induced neuronal apoptosis, and in the neuroprotection caused by rutin.

Several studies have reported deficits in learning and memory in rodents after neonatal exposure to volatile anesthetics $(2,3)$. Working memory refers to cognitive functions that provide synchronized temporary storage and manipulation of the information required to perform intricate cognitive tasks. Working memory is involved in higher cognitive functioning such as planning and sequential behavior. Any deficits in the working memory are directly associated with deficits in behavioral flexibility, and learning deficits have been shown to be associated with hippocampal damage (47).

The Morris water maze test was selected to evaluate the cognitive behavior of rat pups as it is a reliable measure of hippocampus-dependent spatial navigation and reference memory (48). The escape latencies demonstrated by the rats that received isoflurane was significantly longer compared with the controls when assessed $\sim 4$ weeks after the administration of isoflurane. The observations of the present study are suggestive of the possibility of impairments with respect to long-term memory caused by exposure to isoflurane. This could be attributed to the observed isoflurane-induced neurodegeneration. Jevtovic-Todorovic et al (2) reported that, on P7, rats are extremely sensitive to neurotoxic challenge. Thus, any decrease in neural cell proliferation in neonates may eventually lead to cognitive dysfunction by disrupting the neural architecture of 
the hippocampus during a critical period of development, or by depleting the pool of precursor cells present throughout the duration of the animal's life (49).

The marked reduction in neuronal apoptosis as a result of treatment with rutin may have contributed to the significant improvements observed in the performance of the rats in the Morris water maze tests when comparing the isoflurane-only and rutin-treated groups. This may also improve the working memory of the rats.

In conclusion, rutin effectively reduced neuronal apoptosis by regulating JNK/ERK/p38MAPK signalling pathways and modulating the expression of apoptotic proteins. Thus, additional exploration of the effects of rutin is warranted to identify its molecular targets, which may elucidate anesthetic-induced neuronal toxicities.

\section{References}

1. Istaphanous GK and Loepke AW: General anesthetics and the developing brain. Curr Opin Anesthesiol 22: 368-373, 2009.

2. Jevtovic-Todorovic V, Hartman RE, Izumi Y, Benshoff ND, Dikranian K, Zorumski CF, Olney JW and Wozniak DF: Early exposure to common anesthetic agents causes widespread neurodegeneration in the developing rat brain and persistent learning deficits. J Neurosci 23: 876-882, 2003.

3. Satomoto M, Satoh Y, Terui K, Miyao H, Takishima K, Ito M and Imaki J: Neonatal exposure to sevoflurane induces abnormal social behaviors and deficitsin fear conditioning in mice. Anesthesiology 110: 628-637, 2009.

4. Brambrink AM, Evers AS, Avidan MS, Farber NB, Smith DJ, Zhang X, Dissen GA, Creeley CE and Olney JW: Isoflurane-induced neuroapoptosis in the neonatal rhesus macaque brain. Anesthesiology 112: 834-841, 2010.

5. Kong F, Xu L, He D, Zhang X and Lu H: Effects of gestational isoflurane exposure on postnatal memory and learning in rats. Eur J Pharmacol 670: 168-174, 2011.

6. Li Y, Liu C, Zhao Y, Hu K, Zhang J, Zeng M, Luo T, Jiang W and Wang H: Sevoflurane induces short-term changes in proteins in the cerebral cortices of developing rats. Acta Anaesthesiol Scand 57: 380-390, 2013.

7. Li Y, Wang F, Liu C, Zeng M, Han X, Luo T, Jiang W, Xu J and Wang H: JNK pathway may be involved in isoflurane-induced apoptosis in the hippocampi of neonatal rats. Neurosci Lett 545: $17-22,2013$

8. DiMaggio C, Sun LS and Li G: Early childhood exposure to anesthesia and risk of developmental and behavioral disorders in a sibling birth cohort. Anesth Analg 113: 1143-1151, 2011.

9. Ing C, DiMaggio C, Whitehouse A, Hegarty MK, Brady J, von Ungern-Sternberg BS, Davidson A, Wood AJ, Li G and Sun LS: Long-term differences in language and cognitive function after childhood exposure to anesthesia. Pediatrics 130: e476-e485, 2012.

10. Zhao Y, Liang G, Chen Q, Joseph DJ, Meng Q, Eckenhoff RG, Eckenhoff MF and Wei H: Anesthetic-induced neurodegeneration mediated via inositol 1,4,5-trisphosphate receptors. J Pharmacol Exp Ther 333: 14-22, 2010.

11. Zhao YL, Xiang Q, Shi QY, Li SY, Tan L, Wang JT, Jin XG and Luo AL: GABA ergic excitotoxicity injury of the immature hippocampal pyramidal neurons' exposure to isoflurane. Anesth Analg 113: 1152-1160, 2011.

12. Wei H, Kang B, Wei W, Liang G, Meng QC, Li Y and Eckenhoff RG: Isoflurane and sevoflurane affect cell survival and BCL-2/BAX ratio differently. Brain Res 1037: 139-147, 2005.

13. Yon JH, Daniel-Johnson J, Carter LB and Jevtovic-Todorovic V: Anesthesia induces neuronal cell death in the developing rat brain via the intrinsic and extrinsic apoptotic pathways. Neuroscience 135: 815-827, 2005.

14. Harper SJ and Wilkie N: MAPKs: New targets for neurodegeneration. Expert Opin Ther Targets 7: 187-200, 2003.

15. Kaminska B, Gozdz A, Zawadzka M, Ellert-Miklaszewska A and Lipko M: MAPK signal transduction underlying brain inflammation and gliosis as therapeutic target. Anat Rec (Hoboken) 292: 1902-1913, 2009

16. Ji RR, Gereau RW IV, Malcangio M and Strichartz GR: MAP kinase and pain. Brain Res Rev 60: 135-148, 2009.
17. Mousa A and Bakhiet M: Role of cytokine signaling during nervous system development. Int J Mol Sci 14: 13931-13957, 2013.

18. Behrens A, Sibilia M and Wagner EF: Amino-terminal phosphorylation of c-Jun regulates stress-induced apoptosis and cellular proliferation. Nat Genet 21: 326-329, 1999.

19. Jeong HS, Choi HY, Choi TW, Kim BW, Kim JH, Lee ER and Cho SG: Differential regulation of the antiapoptotic action of B-cell lymphoma 2 (Bcl-2) and B-cell lymphoma extra-long (Bcl-xL) by c-Jun N-terminal protein kinase (JNK) 1-involved pathway in neuroglioma cells. Biol Pharm Bull 31: 1686-1690, 2008.

20. Chu R, Upreti M, Ding WX, Yin XM and Chambers TC: Regulation of Bax by c-Jun NH2-terminal kinase and Bcl-xL in vinblastine-induced apoptosis. Biochem Pharmacol 78: 241-248, 2009.

21. Wang WY, Yang R, Hu SF, Wang H, Ma ZW and Lu Y: N-stearoyl-1-tyrosine ameliorates sevoflurane induced neuroapoptosis via MEK/ERK1/2MAPK signaling pathway in the developing brain. Neurosci Lett 541: 167-172, 2013.

22. Sanders RD, Sun P, Patel S, Li M, Maze M and Ma D: Dexmedetomidine provides cortical neuroprotection: Impact on anaesthetic-induced neuroapoptosisin the rat developing brain. Acta Anaesthesiol Scand 54: 710-716, 2010.

23. Metodiewa D, Kochman A and Karolczak S: Evidence for antiradical and antioxidant properties of four biologically active $\mathrm{N}$, N-diethylaminoethyl ethers of flavaone oximes: A comparison with natural polyphenolic flavonoid (rutin) action. Biochem Mol Biol Int 41: 1067-1075, 1997.

24. Jung CH, Lee JY, Cho CH and Kim CJ: Anti-asthmatic action of quercetin and rutin in conscious guinea-pigs challenged with aerosolized ovalbumin. Arch Pharm Res 30: 1599-1607, 2007.

25. Santos KF, Oliveira TT, Nagem TJ, Pinto AS and Oliveira MG: Hypolipidaemic effects of naringenin, rutin, nicotinic acid and their associations. Pharmacol Res 40: 493-496, 1999.

26. Garber JC: Committee for the Update of the Guide for the Care and Use of Laboratory Animals. Guide for the Care and Use of Laboratory Animals, 8th ed. National Academy of Sciences, 2011.

27. Orliaguet G, Vivien B, Langeron O, Bouhemad B, Coriat P and Riou B: Minimum alveolar concentration of volatile anesthetics in rats during postnatal maturation. Anesthesiology 95: 734-739, 2001.

28. Li Y, Liang G, Wang S, Meng Q, Wang Q and Wei H: Effect of fetal exposure to isoflurane on postnatal memory and learning in rats. Neuropharmacology 53: 942-950, 2007.

29. Thornberry NA and Lazebnik Y: Caspases: Enemies within. Science 281: 1312-1316, 1998.

30. Zimmermann KC and Green DR: How cells die: Apoptosis pathways. J Allergy Clin Immnol 108 (4 Suppl): S99-S103, 2001.

31. Vorhees CV and Williams MT: Morris water maze: Procedures for assessing spatial and related forms of learning and memory. Nat Protoc 1: 848-858, 2006.

32. Liang G, Ward C, Peng J, Zhao Y, Huang B and Wei H: Isoflurane causes greater neurodegeneration than an equivalent exposure of sevoflurane in the developing brain of neonatal mice. Anesthesiology 112: 1325-1334, 2010.

33. Istaphanous GK, Howard J, Nan X, Hughes EA, McCann JC, McAuliffe JJ, Danzer SC and Loepke AW: Comparison of the neuroapoptotic properties of equipotent anesthetic concentrations of desflurane, isoflurane, or sevoflurane in neonatal mice. Anesthesiology 114: 578-587, 2011.

34. Oppenheim RW: Cell death during development of the nervous system. Annu Rev Neurosci 14: 453-501, 1991.

35. Rakic S and Zecevic N: Programmed cell death in the developing human telencephalon. Eur J Neurosci 12: 2721-2734, 2000.

36. Blomgren K, Leist M and Groc L: Pathological apoptosis in the developing brain. Apoptosis 12: 993-1010, 2007.

37. Loepke AW and Soriano SG: An assessment of the effects of general anesthetics on developing brain structure and neurocognitive function. Anesth Analg 106: 1681-1707, 2008.

38. Sanders RD, Xu J, Shu Y, Januszewski A, Halder S, Fidalgo A, Sun P, Hossain M, Ma D and Maze M: Dexmedetomidine attenuates isoflurane-induced neurocognitive impairment in neonatal rats. Anesthesiology 110: 1077-1085, 2009.

39. Madsen TM, Kristjansen PE, Bolwig TG and Wörtwein G: Arrested neuronal proliferation and impaired hippocampal function following fractionated brain irradiation in the adult rat. Neuroscience 119: 635-642, 2003.

40. Rola R, Raber J, Rizk A, Otsuka S, VandenBerg SR, Morhardt DR and Fike JR: Radiation-induced impairment of hippocampal neurogenesis is associated with cognitive deficits in young mice. Exp Neurol 188: 316-330, 2004. 
41. Gown AM and Willingham MC: Improved detection of apoptotic cells in archival paraffin sections: Immunohistochemistry using antibodies to cleaved caspase 3. J Histochem Cytochem 50: 449-454, 2002.

42. Zhao H, Yenari MA, Cheng D, Sapolsky RM and Steinberg GK: $\mathrm{Bcl}-2$ overexpression protects against neuron loss within the ischemic margin following experimental stroke and inhibits cytochrome $c$ translocation and caspase-3 activity. J Neurochem 85 1026-1036, 2003

43. Guan QH, Pei DS, Zhang QG, Hao ZB, Xu TL and Zhang GY: The neuroprotective action of SP600125, a new inhibitor of JNK, on transient brain ischemia/reperfusion-induced neuronal death in rat hippocampal CA1 via nuclear and non-nuclear pathways. Brain Res 1035: 51-59, 2005.

44. Guan QH,Pei DS, Zong YY,Xu TL and Zhang GY: Neuroprotection against ischemic brain injury by a small peptide inhibitor of c-Jun N-terminal kinase (JNK) via nuclear and non-nuclear pathways. Neuroscience 139: 609-627, 2006.
45. Han JY, Jeong EY, Kim YS, Roh GS, Kim HJ, Kang SS, Cho GJ and Choi WS: C-jun N-terminal kinase regulates the interaction between 14-3-3 and Bad in ethanol-induced cell death. J Neurosci Res 86: 3221-3229, 2008.

46. Zheng S and ZuoZ: Isoflurane preconditioning induces neuroprotection against ischemia via activation of P38 mitogen-activated protein kinases. Mol Pharmacol 65: 1172-1180, 2004.

47. Morris RG, Garrud P, Rawlins JN and O'Keefe J: Place navigation impaired in rats with hippocampal lesions. Nature 297: 681-683, 1982.

48. D'Hooge R and De Deyn PP: Applications of the Morris water maze in the study of learning and memory. Brain Res Brain Res Rev 36: 60-90, 2001.

49. Sall JW, Stratmann G, Leong J, McKleroy W, Mason D, Shenoy S, Pleasure SJ and Bickler PE: Isoflurane inhibits growth but does not cause cell death in hippocampal neural precursor cells grown in culture. Anesthesiology 110: 826-833, 2009. 